\title{
THE NUMERICAL SOLUTION OF THE NAVIER-STOKES EQUATIONS FOR AN INCOMPRESSIBLE FLUID ${ }^{1}$
}

\author{
BY ALEXANDRE JOEL CHORIN
}

Communicated by E. Isaacson, June 21, 1967

A finite difference method for solving the Navier-Stokes equations for an incompressible fluid has been developed. This method uses the primitive variables, i.e. the velocities and the pressure, and is equally applicable to problems in two and three space dimensions. Essentially it constitutes an extension to time dependent problems of the artificial compressibility method introduced in [1] for steady flow problems.

The equations to be solved can be written in the dimensionless form

$$
\begin{aligned}
\partial_{t} u_{i}+R u_{j} \partial_{j} u_{i} & =-\partial_{i} p+\Delta u_{i}+E_{i}, \quad \Delta \equiv \sum \partial_{j}^{2}, \\
\partial_{j} u_{j} & =0
\end{aligned}
$$

where $u_{i}$ are the velocity components, $E_{i}$ the external forces, $p$ is the pressure, and $R$ the Reynolds number. $\partial_{i}$ denotes differentiation with respect to the space variable $x_{i}$, and $\partial_{t}$ differentiation with respect to the time $t$. The summation convention is used in (1) and (2). The equations are to be solved in a domain D. Problems where the velocities or their derivatives are prescribed at the boundary or where some boundary conditions are replaced by periodicity conditions have been investigated. For simplicity, we shall describe the case where the velocities are given at the boundary and shall assume that a cartesian coordinate system is used. An important feature of the method is the use of equation (2) rather than a derived equation (see e.g. [2]) for determining the pressure. This makes it possible to satisfy the equation of continuity at the boundary and determine the pressure in a natural way.

Let $D u=0$ be a difference approximation to $\partial_{j} u_{j}=0 . D u$ takes a different form in the interior of $D$ where first order centered differences are used, and at the boundary where one-sided differences up to second order are used to insure second order accuracy. It is assumed that at the time $t=n \Delta t$, velocity and pressure fields $u_{i}^{n}$ and $p^{n}$, where $u^{n} \equiv u(n \Delta t)$ and $p^{n} \equiv(n \Delta t)$, are given, satisfying $D u^{n}=0$. The task at hand is to compute $u^{n+1}, p^{n+1}$ from equation (1), so that $D u^{n+1}=0$.

Auxiliary fields $u_{i}^{\text {aux }}$ are first computed through

1 This work was partially supported by the U. S. Atomic Energy Commission, Contract No. AT(30-1)-1480. 


$$
u_{i}^{\mathrm{aux}}=u_{i}^{n}+\Delta t F_{i} u
$$

where $F_{i} u$ approximates

$$
-R u_{j} \partial_{j} u_{i}+\Delta u_{i}+E_{i}, \quad \Delta \equiv \sum \partial_{j}^{2} .
$$

$F_{i} u$ may depend on $u_{i}^{\text {aux }}, u_{i}^{n}$, and intermediate fields, say $u^{*}, u^{* *}$, etc. In general, an implicit alternating direction scheme is used to find the fields $u^{\text {aux }}, u^{*}$, etc. One of several versions of that method is the following (see Samarski [3]): Let $\Delta x_{i}, i=1,2,3$, be the space variable increments, let $u_{i(q, r, s)}$ denote $u_{i}\left(q \Delta x_{1}, r \Delta x_{2}, s \Delta x_{3}\right)$, and let $E_{i(q, r, s)}$ denote $E_{i}\left(q \Delta x_{1}, r \Delta x_{2}, s \Delta x_{3}\right), u_{1}^{\text {aux }}$ is then computed through

$$
\begin{aligned}
u_{1(q, r, s)}^{*}= & u_{1(q, r, s)}^{n}-R \frac{\Delta t}{2 \Delta x_{1}} u_{1(q, r, s)}^{n}\left(u_{1(q+1, r, s)}^{*}-u_{1(q-1, r, s)}^{*}\right) \\
& +\frac{\Delta t}{\Delta x_{1}^{2}}\left(u_{1(q+1, r, s)}^{*}+u_{1(q-1, r, s)}^{*}-2 u_{1(q, r, s)}^{*},\right. \\
\stackrel{u_{1(q, r, s)}^{* *}=}{*} & u_{1(q, r, s)}^{*}-R \frac{\Delta t}{2 \Delta x_{2}} u_{2(q, r, s)}^{*}\left(u_{1(q, r+1, s)}^{* *}-u_{1(q, r-1, s)}^{* *}\right) \\
& +\frac{\Delta t}{\Delta x_{2}^{2}}\left(u_{1(q, r+1, s)}^{* *}+u_{1(q, r-1, s)}^{* *}-2 u_{1(q, r, s)}^{* *},\right. \\
u_{1(q, r, s)}^{\operatorname{aux}}= & u_{1(q, r, s)}^{* *}-R \frac{\Delta t}{2 \Delta x_{3}} u_{(q, r, s)}^{* *}\left(u_{1(q, r, s+1)}^{\operatorname{aux}}-u_{1(q, r, s-1)}^{\operatorname{aux}}\right) \\
& +\frac{\Delta t}{\Delta x_{3}^{2}}\left(u_{1(q, r, s+1)}^{\operatorname{aux}}+u_{1(q, r, s-1)}^{\operatorname{aux}}-2 u_{1(q, r, s)}^{\mathrm{aux}}\right) \\
& +\Delta t E_{1(q, r, s),}
\end{aligned}
$$

with similar expressions for $u_{2}^{\text {aux }}, u_{3}^{\text {aux }}$.

So far the pressure term in (1) and equation (2) have not been taken into account. An iteration procedure is now introduced to find $u_{i}^{n+1}$ inside $D$ and $p^{n+1}$ in $D$ and on its boundary by setting

$$
\begin{aligned}
& p^{n+1,1}=p^{n}, \\
& p^{n+1, m+1}-p^{n+1, m}=-\lambda D u^{n+1, m+1}, \\
& u_{i}^{n+1, m+1}=u_{i}^{\text {aux }}-\Delta t G_{i}^{m} p \quad(m \geqq 1)
\end{aligned}
$$

where $\lambda$ is a parameter, the quantities $u_{t}^{n+1, m}$ and $p^{n+1, m}$ are successive approximations to $u_{i}^{n+1}$ and $p^{n+1}$, and $G_{i}^{m} p$ is a function of $p^{n+1, m+1}$ and $p^{n+1, m}$ which tends to a difference form of $\partial_{i} p^{n+1}$ as $\left|p^{n+1, m+1}-p^{n+1, m}\right|$ tends to zero. The form of $G_{i}^{m} p$ is crucial for the accuracy and rapid 
convergence of the method and will be defined below in equations (5a) and (5b).

When for some $l$ and some small predetermined constant $\epsilon$

we define

$$
\max _{D}\left|p^{n+1, l+1}-p^{n+1, l}\right| \leqq \epsilon
$$

$$
u_{i}^{n+1}=u_{i}^{n+1, l+1}, \quad p^{n+1}=p^{n+1, l+1} .
$$

If the velocity component $u_{i}$ is prescribed at the boundary, $u_{i}^{n+1, m+1}$ and $u_{i}^{\text {aux }}$ in (4b) and (4c) are replaced by the given value of $u_{i}$. The iterations (4b) and (4c) insure that equation (1) is satisfied inside the domain $D$ and equation (2) is satisfied in $D$ and at the boundary.

The iteration (4) is carried out as follows: Let $p_{(q, r, s)}$ denote $p\left(q \Delta x_{1}, r \Delta x_{2}, s \Delta x_{3}\right)$.

$$
p_{(q, r, s)}^{n+1, m+1}, \begin{gathered}
n+1, m+1 \\
u_{1(q \pm 1, r, s)},
\end{gathered} u_{2(q, r \pm 1, s)}^{n+1, m+1}, u_{3(q, r, s \pm 1)}^{n+1, m+1}
$$

are to be evaluated simultaneously; $p_{a, r, s}^{n+1, m+1}$ using equation (4b); $u_{1(q \pm 1, r, s)}^{n+1, m+1}$ using the formulas

$$
\begin{aligned}
& u_{1(q+1, r, s)}^{n+1, m+1}=u_{1(q+1, r, s)}^{\mathrm{aux}}-\frac{\Delta t}{2 \Delta x_{1}}\left(p_{(q+2, r, s)}^{n+1, m}-\frac{1}{2}\left(p_{(q, r, s)}^{n+1, m+1}+p_{(q, r, s)}^{n+1, m}\right)\right) \\
& u_{1(q-1, r, s)}^{n+1, m+1}=u_{1(q-1, r, s)}^{\mathrm{aux}}-\frac{\Delta t}{2 \Delta x_{1}}\left(\frac{1}{2}\left(p_{(q, r, s)}^{n+1, m+1}+p_{(q, r, s)}^{n+1, m}\right)-p_{(q-2, r, s)}^{n+1, m}\right)
\end{aligned}
$$

with similar expressions for $u_{2}, u_{3}$. Natural modifications of these expressions are introduced near the boundary, where some of the $u_{i}$ are prescribed and where $D u$ employs noncentered differences. From (4b), (5a), (5b) and the analogous expressions for $u_{2}$ and $u_{3}$, we obtain a system of seven equations in the seven unknowns

$$
\begin{gathered}
n+1, m+1 \\
u_{1(q \pm 1, r, s)}^{n+1, m+1},
\end{gathered}, u_{2(q, r \pm 1, s)}^{n+1}, u_{3(q, r, s \pm 1)}^{n+1, m+1} \text { and } p_{(q, r, s)}^{n+1, m+1}
$$

which can be solved explicitly. (There is no need to evaluate the $u_{i}^{n+1, m+1}$ until after the $p^{n+1, m}$ have converged, since the $u_{i}^{n+1, m+1}$ can be eliminated from the seven equations.)

The resulting iteration procedure converges for all $\lambda>0$ under natural restrictions on the domain $\mathcal{D}$, and a best value of $\lambda$, say $\lambda_{\text {opt }}$, can be determined. For this iteration scheme it can be expected that $\epsilon=O(\Delta t)$ is sufficient to insure the overall accuracy of the scheme to 
$O(\Delta t), 0 \leqq t \leqq T$. This results in a reduction of the amount of computational labor. It is assumed that $\Delta t=O\left(\Delta x^{2}\right)$.

That the scheme is indeed accurate to $O(\Delta t)$ has been verified on certain simple test problems for which an analytical solution can be obtained. In one such problem, used as test problem by Pearson [4] for a vorticity-stream function method, $D$ is a two dimensional square $0 \leqq x_{1} \leqq \pi, 0 \leqq x_{2} \leqq \pi . R=1, E_{1}=E_{2}=0$, and the exact solution is

$$
\begin{gathered}
u_{1}=-\cos x_{1} \sin x_{2} e^{-2 t}, \quad u_{2}=\sin x_{1} \cos x_{2} e^{-2 t}, \\
p=-\frac{1}{4}\left(\cos 2 x_{1}+\cos 2 x_{2}\right) e^{-4 t}
\end{gathered}
$$

where appropriate boundary conditions and initial data are used. Accurate results have been obtained at the price of a modest amount of computing effort. For $\Delta x_{1}=\Delta x_{2}=\pi / 19=\Delta x, \Delta t / \Delta x^{2}=2$, the maximum relative error in $u_{1}, u_{2}$ over $D$ after one step is less than $0.08 \%$; and after sixteen steps, less than $0.02 \%$, using $\lambda=\lambda_{\text {opt }}$ $=\Delta x^{2} /(\Delta t \sin 2 \Delta x), \epsilon=\Delta x^{3} ;$ the largest $l$ needed is 6 .

The method is presently being applied to the problem of wave number selection and finite amplitude stability in a convective layer (the Benard problem, see e.g. [5]) and to a numerical study of energy cascading between large and small eddies in a model of turbulence (the Green-Taylor problem [6]). The results of these studies and further details on the numerical method will be presented in a forthcoming paper and will point out crucial differences between the behavior of solutions of the Navier-Stokes equations in two and three space dimensions.

\section{BIBLIOGRAPHY}

1. A. J. Chorin, A numerical method for solving incompressible viscous flow problems, J. Comp. Physics (to appear).

2. F. H. Harlow and J. E. Welch, Numerical calculations of time dependent viscous incompressible flow of fuid with a free surface, Phys. Fluids 8 (1965), 2182.

3. A. A. Samarski, On an economical difference method for the solution of a multidimensional parabolic problem in an arbitrary region, U.S.S.R. Comput. Math. and Math. Phys. 5 (1963), 894.

4. C. E. Pearson, A computational method for time dependent two dimensional incompressible viscous flow problems, Sperry-Rand Research Center, Sudbury, Mass., Report No. SRRC-RR-64-17 (1964).

5. A. J. Chorin, Numerical study of thermal convection in a fuid layer heated from below, AEC Report No. NYO-1480-61, New York University (1966).

6. G. I. Taylor and A. E. Green, Mechanism of the production of small eddies fron. large ones, Proc. Roy. Soc. A 158 (1937), 499.

Courant Institute of Mathematical Sciences, New York University 